\title{
Large-amplitude internal tides, solitary waves, and turbulence in the central Bay of Biscay
}

\author{
X. H. Xie,${ }^{1}$ Y. Cuypers, ${ }^{1}$ P. Bouruet-Aubertot, ${ }^{1}$ B. Ferron, ${ }^{2}$ A. Pichon, ${ }^{3}$ A. Lourenço, ${ }^{1}$ \\ and N. Cortes ${ }^{2}$ \\ Received 28 February 2013; revised 3 May 2013; accepted 3 May 2013; published 7 June 2013.
}

[1] Microstructure and fine-scale measurements collected in the central Bay of Biscay during the MOUTON experiment are analyzed to investigate the dynamics of internal waves and associated mixing. Large-amplitude internal tides (ITs) that excite internal solitary waves (ISWs) in the thermocline are observed. ITs are dominated by modes 3 and 4, while ISWs projected on mode 1 that is trapped in the thermocline. Therein, ITs generate a persistent narrow shear band, which is strongly correlated with the enhanced dissipation rate in the thermocline. This strong dissipation rate is further reinforced in the presence of ISWs. Dissipation rates during the period without ISWs largely agree with the MacKinnonGregg scaling proposed for internal wavefields dominated by a low-frequency mode, while they show poor agreement with the Gregg-Henyey parameterization valid for internal wavefields close to the Garrett-Munk model. The agreement with the MacKinnon-Gregg scaling is consistent with the fact that turbulent mixing here is driven by the lowfrequency internal tidal shear. Citation: Xie, X. H., Y. Cuypers, P. Bouruet-Aubertot, B. Ferron, A. Pichon, A. Lourenço, and N. Cortes (2013), Large-amplitude internal tides, solitary waves, and turbulence in the central Bay of Biscay, Geophys. Res. Lett., 40, 2748-2754, doi:10.1002/grl.50533.

\section{Introduction}

[2] It is well known that the Bay of Biscay (BB) is a marginal sea with large-amplitude internal tides (ITs). Therein, ITs are mainly generated on the slope via interaction between the barotropic tide and the steep topography [e.g., Pingree and New, 1991; Gerkema et al., 2004; Pichon et al., 2013]. Once generated, they propagate both seaward and shoreward. Linear internal wave theory and observations show that the seaward traveling ITs propagate along a beam or ray path with a slope to the horizontal given by

$$
\alpha= \pm\left[\left(\omega^{2}-f^{2}\right) /\left(N^{2}-\omega^{2}\right)\right]^{1 / 2},
$$

where $\omega$ is the semidiurnal frequency, $f$ is the local inertial frequency, and $N$ is the buoyancy frequency [Pingree and New, 1991]. The theory also predicts that the offshore propagating beam first reflects at the bottom and next at the sea surface in the central BB (Figure 1a) [Gerkema et al.,

${ }^{1}$ LOCEAN, UMR 7167, Université Pierre et Marie Curie, Paris, France.

${ }^{2}$ LPO, UMR 6523, CNRS-IFREMER-IRD-UBO, Brest, France.

${ }^{3}$ Centre Militaire d'Océanographie, SHOM, Brest, France.

Corresponding author: X. H. Xie, State Key Laboratory of Tropical Oceanography, South China Sea Institute of Oceanology, Chinese Academy of Sciences, Guangzhou 510301, China. (xhxie@scsio.ac.cn)

C2013. American Geophysical Union. All Rights Reserved. 0094-8276/13/10.1002/grl.50533
2004; Pichon et al., 2013]. Using temperature and velocity records obtained from the central BB, New and Pingree [1990] observed large internal interfacial tides and internal solitary waves (ISWs) in the thermocline. These waves were as large as those observed at the shelf edge, and they were thus conjectured to be locally aroused in the central BB, where internal tidal beams hit the seasonal thermocline near the sea surface. This hypothesis was confirmed by satellite imagery [New and DaSilva, 2002]. Theoretical framework for this process was given by Gerkema [2001, hereafter referred as to G01] who showed that scattering of IT beams in the thermocline leads to the local generation of large ITs and ISWs. This was seen in numerical models [Grisouard et al., 2011] and reproduced in laboratory experiments [Mercier et al., 2012].

[3] While there are numerous observations of ITs and ISWs in the central BB, there have never been measurements in the central BB allowing a fine characterization of ITs and ISWs. Another aspect that has never been quantified is the turbulent mixing induced by these internal waves. In this paper, we report on joint fine-scale and microstructure measurements performed for the first time in the central BB. We first characterize vertical structures of ITs and ISWs and next dissipation rate $(\varepsilon)$ of turbulent kinetic energy and turbulent mixing induced by ITs and ISWs. Finally, fine-scale parameterizations are tested against microstructure measurements.

\section{Data}

[4] Data were collected at a location in the central BB $\left(45.75^{\circ} \mathrm{N}, 7.38^{\circ} \mathrm{W}\right)$ during the MOUTON experiment in summer 2008, where the water depth is about $4800 \mathrm{~m}$ (Figure 1a). This location is about $200 \mathrm{~km}$ away from generation area of internal tidal beams on the northern slope of BB (Figure 1a) [Pichon et al., 2013]. From 18 September 01(h):10(min) to 19 September 04:00, combined conductivity-temperature-depth/ lowered acoustic Doppler current profiler (CTD/LADCP) yo-yoing was performed. This period corresponds to the spring tide which reaches a maximum around 18 September. Sixteen profiles were collected with an effective depth range from 6 to $2663 \mathrm{~m}$. From 04:50 to 21:20 on 19 September, two microstructure instruments, VMP (Vertical Microstructure Profiler) and SCAMP (Self-Contained Autonomous Microstructure Profiler), were alternatively deployed. Dissipation rate estimates with SCAMP are based on Batchelor fitting of the temperature gradient spectrum measured by highfrequency temperature sensors [Ruddick et al., 2000], whereas VMP estimates are based on measurements of microscale vertical shear. Previous studies have shown that the different methods for estimating $\varepsilon$ using these two instruments fall within a factor 2 [Kocsis, et al., 1999]. While 19 VMP profiles 

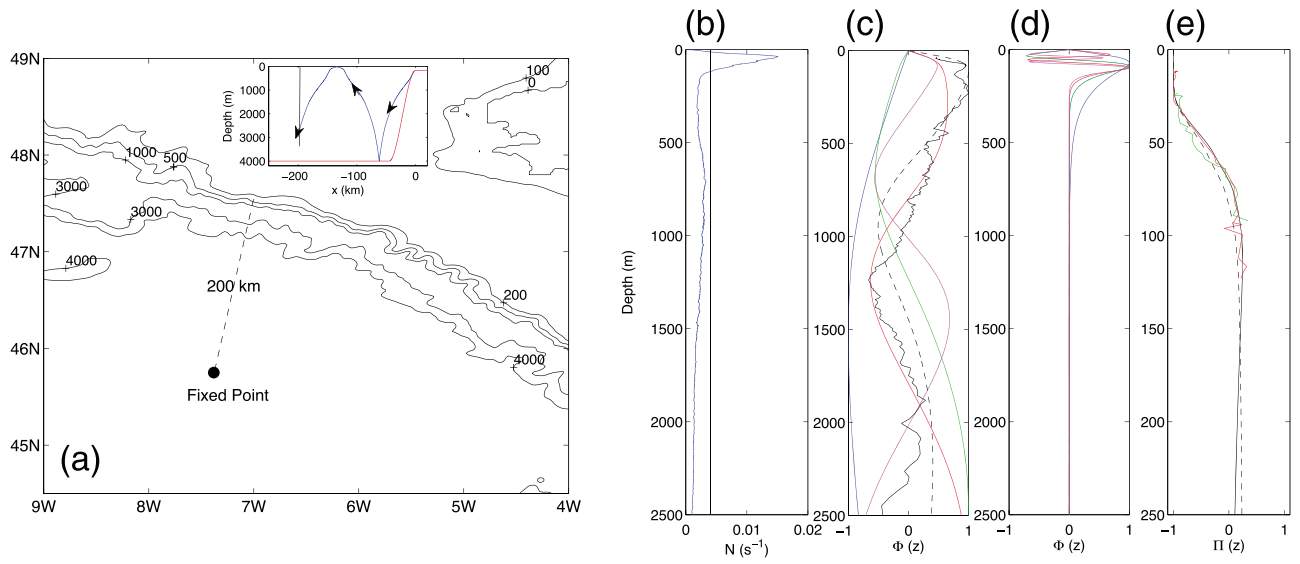

Figure 1. (a) Map showing bathymetry in the BB and the observation site (the black point). The dash line indicates the possible horizontal propagation path of the seaward traveling internal tidal beams generated on the northern slope. Their characteristic path is given in the inserted panel (blue line with arrows). The red curve is typical model topography with horizontal resolution of $100 \mathrm{~m}$ in the $\mathrm{BB}$ along the cross-slope direction. The vertical line indicates the observation location. (b) Profile of $N(z)$ : The vertical line indicates a frequency of $2 \pi /(25 \mathrm{~min})$. Vertical structures of displacement $\Phi(z)$ for mode 1 (blue), mode 2 (green), mode 3 (red), and mode 4 (pink) waves with (c) semidiurnal and (d) $2 \pi /(25$ min) frequencies. In Figure 1c, the black solid line is the measured IT displacement profile around $3 \mathrm{~h} / 19 \mathrm{September}$ (black solid line). The dashed line is sum of projected mode 3 and mode 4. (e) Observed velocity profiles (red and blue lines) during two ISWs (marked by arrows in Figure 3a) in a semi-Lagrangian frame and velocity profiles of IT mode 4 (black dashed line) and trapped mode 1 (black solid line). Note that both displacements and velocities have been normalized.

were obtained from the surface to depths of 400-500 m, 15 SCAMP profiles were collected from the surface to depths of $70-110 \mathrm{~m}$. Data above $10 \mathrm{~m}$ were removed due to the contamination by the ship's wake. Current velocities were inferred from a $150 \mathrm{kHz}$ and a $38 \mathrm{kHz}$ broadband shipboard acoustic Doppler current profilers (ADCPs). Measurement range of the former ADCP is from $18 \mathrm{~m}$ to $162 \mathrm{~m}$ with a $2 \mathrm{~min}$ timeaverage interval and $4 \mathrm{~m}$ bin, and the latter is from $55 \mathrm{~m}$ to $1231 \mathrm{~m}$ with the same time-average interval and $24 \mathrm{~m}$ bin. We used here the $150 \mathrm{kHz}$ ADCP data to compute $4 \mathrm{~m}$ shear $\left(S_{4}\right)$ during microstructure measurements.

[5] CTD data were de-spiked and averaged into $1 \mathrm{~m}$ bins, while $\varepsilon$ is computed from VMP and SCAMP data using $1 \mathrm{~m}$ bins. Since SCAMP was not equipped with conductivity sensors, salinity during SCAMP measurements was estimated by a linear interpolation of VMP salinity data.

\section{Results}

\subsection{Mean Stratification and Semidiurnal IT Raypath}

[6] The time-averaged CTD data were used to compute the buoyancy frequency $N(z)$. The climatological temperature and salinity data at the measurement location obtained from the World Ocean Atlas 2009 were used to complete the profile below $2663 \mathrm{~m}$ depth (Figure $1 \mathrm{~b}$ ). The $N(z)$ profile showing the largest value at $40-70 \mathrm{~m}$ (seasonal thermocline) and a small peak near $800 \mathrm{~m}$ (permanent pycnocline) (Figure 1b) is typical for the central BB during summer [G01]. An internal tidal beam path is computed using $N(z)$ and a typical model topography in the BB. The surface reflection site of internal tidal beams in the central $\mathrm{BB}$ is $\sim 140 \mathrm{~km}$ away from their generation location and $\sim 60 \mathrm{~km}$ away from our observation site (Figure 1a, embedded panel).

\subsection{Internal Interfacial Tides and ISWs}

[7] Time series of temperature show large thermocline depressions with a semidiurnal period, namely, the socalled internal interfacial tides (Figure 2a). Their amplitude from crest to trough is in the range $40-70 \mathrm{~m}$. Note that the largest isotherm displacements are often observed below $1800 \mathrm{~m}$ (not shown). These large displacements are likely induced by the descending beam, which intersects the observational location at large depth (see inserted panel in Figure 1a). During the second observation period, that of VMP/SCAMP measurements, the isotherms slope in the thermocline depressions becomes steeper, implying the enhancement of IT nonlinearity. Although the maximum tidal forcing is on 18 September, nonlinearity is larger on 19 September. This delay may result from the propagation time of ITs from the shelf break to the observational site (i.e., 2-4 days considering the phase speed of the interfacial mode in the seasonal thermocline). As a result of enhanced nonlinearity, high-frequency ISWs are generated in troughs and surface velocities are increased (Figures $2 b$ and $3 a$ ). Note that the averaged time interval between two ISW events is equal to $25 \mathrm{~min}$, and ISWs could not be identified from temperature data due to their low temporal resolution (about 20 min) but only from ADCP data.

[8] We determine in the following the directions of propagation of ITs and ISWs as well as their vertical structure. The large thermocline depressions often cause southward velocity in the surface layer (Figures $2 \mathrm{c}$ and $2 \mathrm{~d}$ ), implying a southward propagation of ITs. These large southward interfacial tides likely result from the scattering of the seaward traveling internal tidal beams generated on the northern slope [G01]. However, a more complex pattern can be observed on 19 September, from 06:00 to 12:00. During this period, two oppositely traveling troughs are observed in the large thermocline depression: the first one generates 

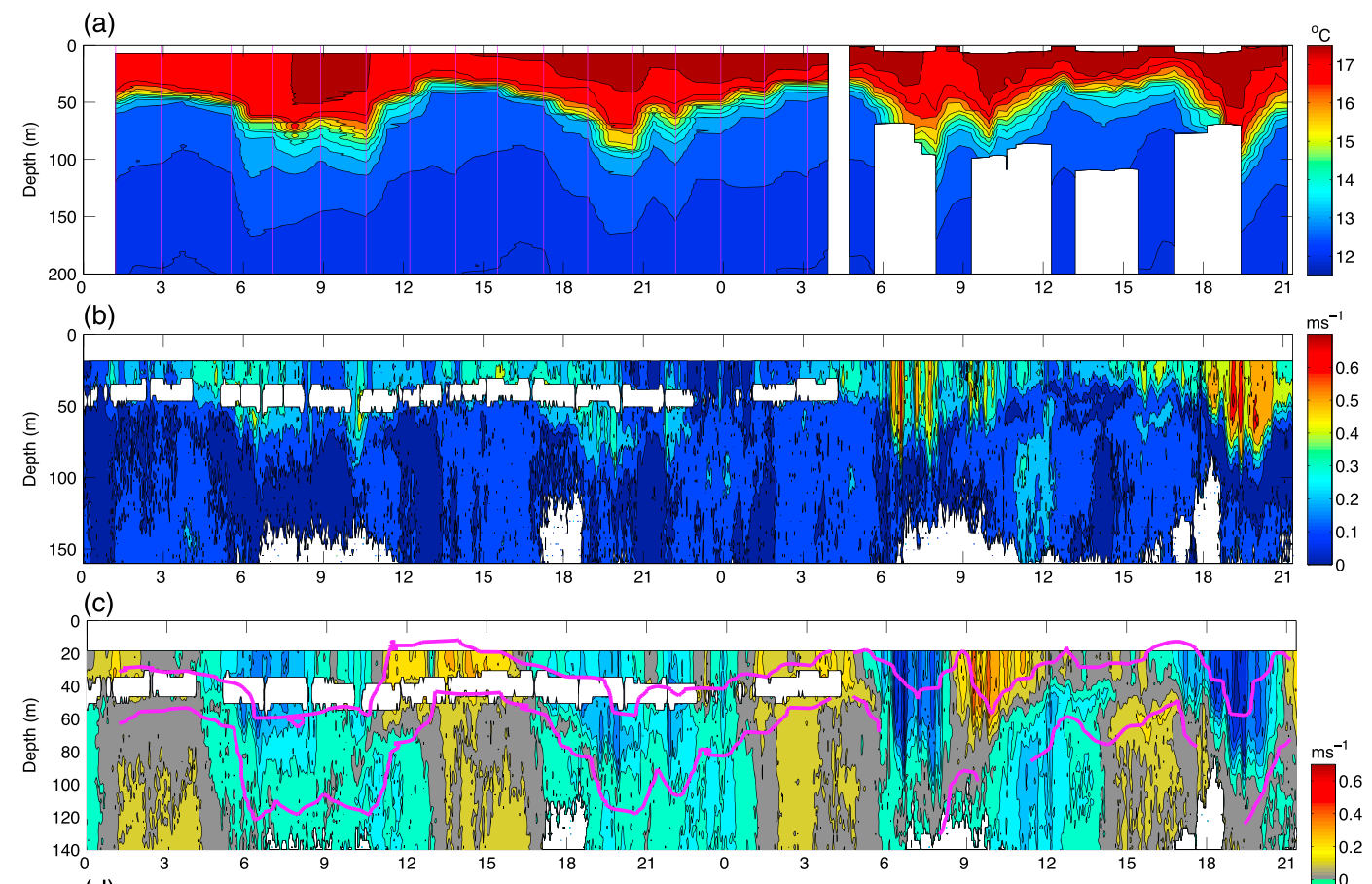

(d)

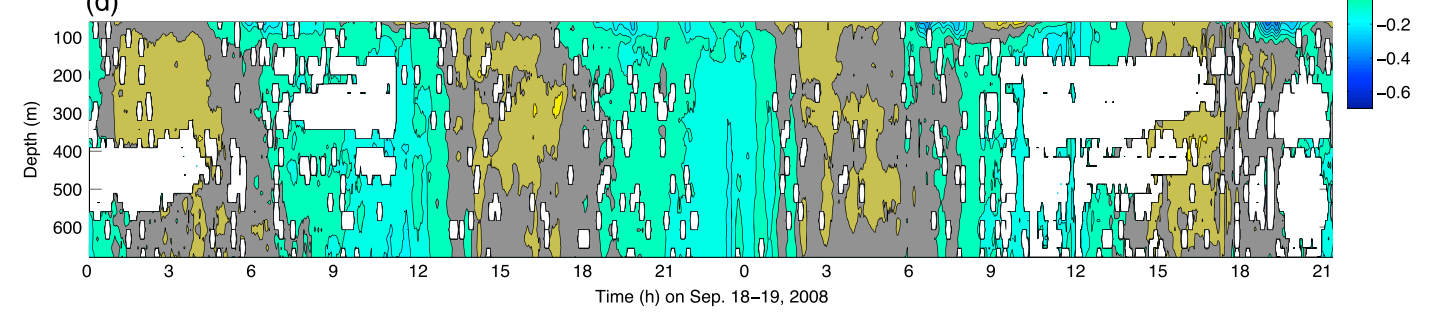

Figure 2. (a) Temperature. For clarity, only the upper $200 \mathrm{~m}$ is shown. Pink vertical lines indicate the times of CTD profiles. (b) Current velocity amplitude. Time series of meridional velocities obtained from (c) $150 \mathrm{kHZ}$ and (d) $38 \mathrm{kHZ}$ ADCPs. A 10 min moving averaged has been applied to the data displayed in Figure 2d. Two pink curves in Figure 2c are isotherms of $17.3^{\circ} \mathrm{C}$ and $12.9^{\circ} \mathrm{C}$, indicating depths of the thermocline, similar for Figures $3 \mathrm{~b}-3 \mathrm{e}$ (black curves in Figures $3 \mathrm{~b}$ and $3 \mathrm{c}$ ).

southward surface velocity while the second one induces northward surface velocity. The trough propagating northward may be associated with internal tidal beams originating from the southern slope [Pichon et al., 2013]. Since the velocity direction caused by ISWs is the same as that of thermocline depressions (Figure 2c), their propagating direction is consistent with that of internal tides.

[9] To investigate the vertical structure of ITs and ISWs, we have computed vertical modal functions of displacement $\Phi(z)$ and horizontal velocity $\Pi(z)$ at semidiurnal and $2 \pi /$ (25 $\mathrm{min}$ ) frequencies following equations 6.10.2, 6.10.3, and 6.10.6 from Gill [1982]. Note that $25 \mathrm{~min}$ is the averaged period of the observed ISWs. Modal structures of waves with these two frequencies show large difference (Figures 1c and 1d), as the high frequency is larger than $N$ below the thermocline (see Figure 1b). As a result, the high-frequency modes are trapped in the thermocline (Figures $1 \mathrm{~d}$ and 2b). To distinguish mode functions of high-frequency waves from that of low-frequency waves, we refer to the former as trapped mode structures and to the latter as IT mode thereafter. In order to infer IT mode structures, we compute time series of isopycnal vertical displacement from CTD measurements. Then we extract the semidiurnal amplitude for each depth using a harmonic analysis of the displacement time series. The resulting normalized displacement profile at the semidiurnal frequency is plotted in Figure 1c. The mode 3 structure roughly reflects depth variation of IT displacement except for depths below $1800 \mathrm{~m}$ (Figure 1g), where other modes may be dominant due to the downward IT beam. A mode projection on IT displacements by the least square method indicates that mode 3 (mode 4 ) explains about $61 \%(23 \%)$ of total potential energy above the depth of $500 \mathrm{~m}$. Accordingly, the sum of the projected mode 3 and mode 4 reproduces well the variation of semidiurnal displacement above $500 \mathrm{~m}$ depth (Figure 1c). Meanwhile, measured velocity profiles when ISWs pass the fixed point are well fitted by trapped mode 1 (Figure 1e). Note that as vertical advection of velocity signals by thermocline displacements is strong (see Figure 2c), Eulerian depth of velocity profiles in Figure 1e was transformed to semi-Lagrangian depth. Interestingly, the vertical structures of IT mode 4 and trapped mode 1 are very similar within the thermocline (Figure 1e), which suggests that the observed ISWs are likely to result from mode 4 ITs.

\subsection{Microstructure Measurements}

[10] Time series of dissipation rates are shown in Figure 3b. The largest $\varepsilon$ values often occur near the surface, which may be directly associated with wind stirring in the mixed layer. 


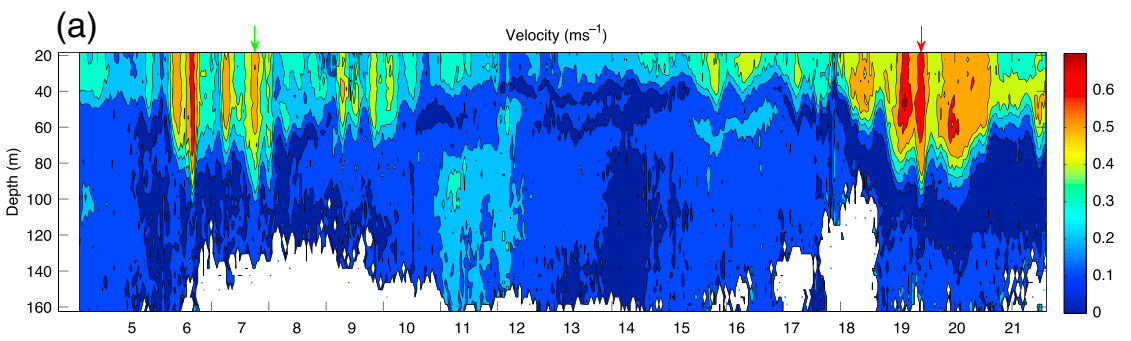

(b)
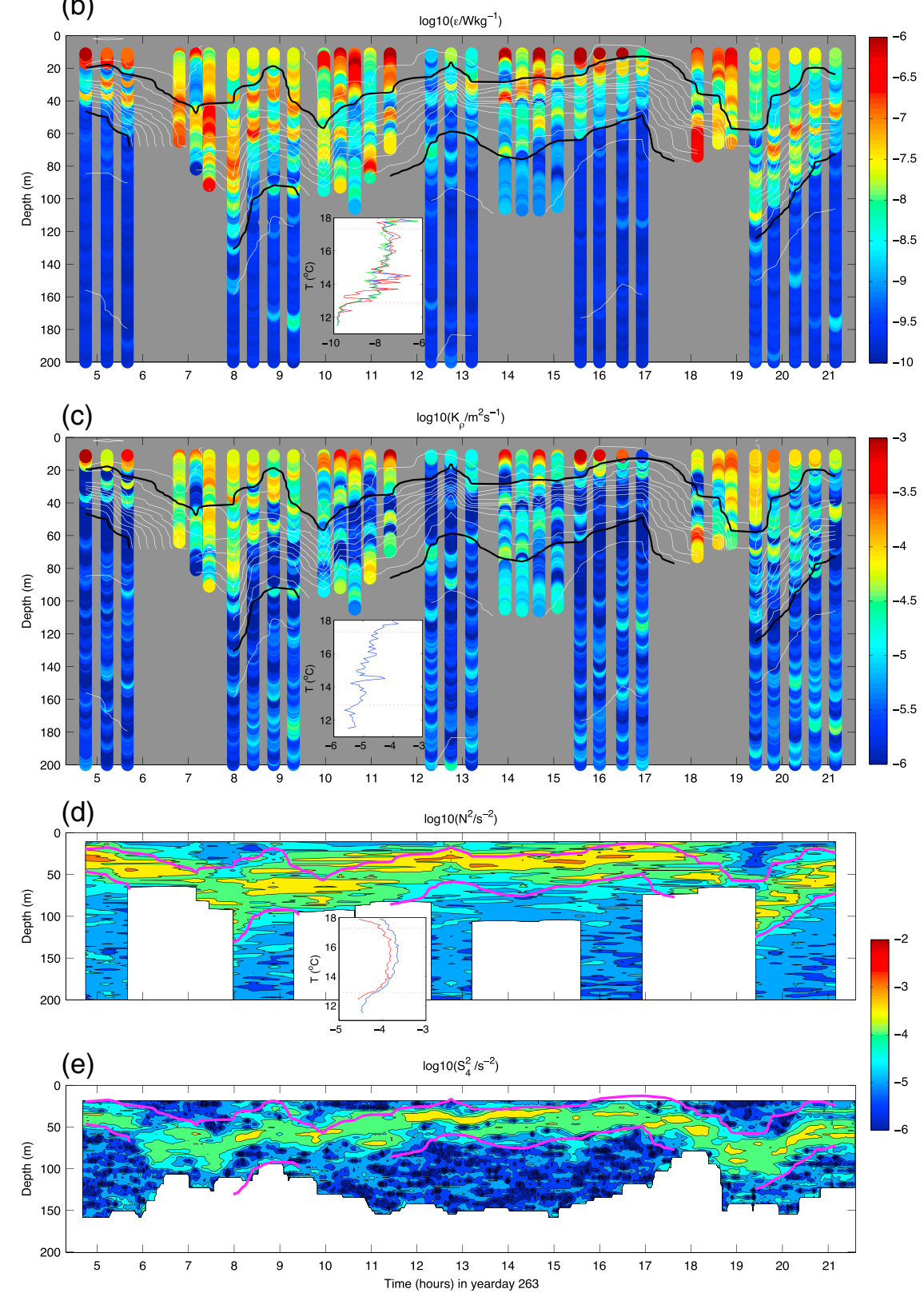

Figure 3. (a) Velocity amplitudes during VMP/SCAMP observation. The arrows indicate ISWs plotted in Figure 2b. (b) Dissipation rate. (c) Diapycnal diffusivity. Isotherms whose interval is $0.5^{\circ} \mathrm{C}$ (white lines) are overplotted on Figures $3 \mathrm{~b}$ and 3c. (d) $N^{2}$. (e) $\left(S_{4}\right)^{2}$. A 4 m running average is used for Figures $3 \mathrm{~b}$ and $3 \mathrm{c}$. Data in Figure 3e have 30 min resolution. The embodied panels in Figures $3 \mathrm{~b}-3 \mathrm{~d}$ is time-averaged $\varepsilon, K_{\rho}$, and $N^{2}$ (blue) and $\left(S_{4}\right)^{2}$ (red) along the isotherms. $\varepsilon$ values in the inserted panel in Figure $3 \mathrm{~b}$ are averaged over all profiles (blue) and profiles near (red) and without (green) ISW events. $K_{\rho}, N^{2}$, and $\left(S_{4}\right)^{2}$ in Figures $3 \mathrm{c}$ and $3 \mathrm{~d}$ are averaged over all profiles.

Enhanced $\varepsilon$ values are also encountered in the thermocline with a mean $\varepsilon$ value of $2.3 \times 10^{-8} \mathrm{~W} \mathrm{~kg}^{-1}$. Diapycnal diffusivity, $K_{\rho}$, was computed according to the relationship established by Shih et al. [2005] who distinguished different regimes as a function of turbulent intensity: $K_{\rho}=\lambda \varepsilon / N^{2}$, where $\lambda=2\left[\varepsilon /\left(v N^{2}\right)\right]^{-1 / 2}\left(v\right.$ is the molecular viscosity) when $\varepsilon /\left(v N^{2}\right)$ $>100$ otherwise $\lambda=0.2$. Enhanced $K_{\rho}$ values also occur in the thermocline (Figure 3c) with a mean value equal to 
(a)

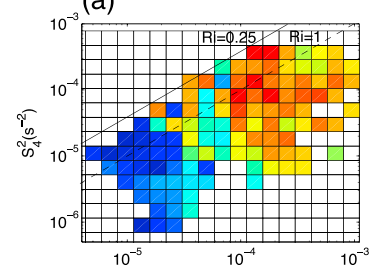

(d)
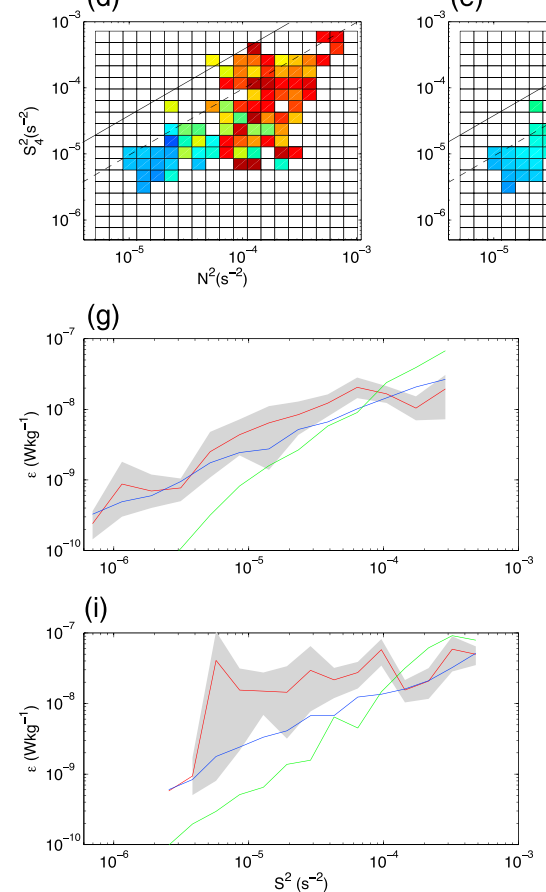

(b)

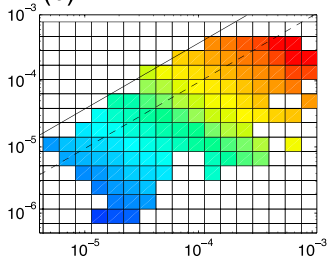

$(\mathrm{e})$

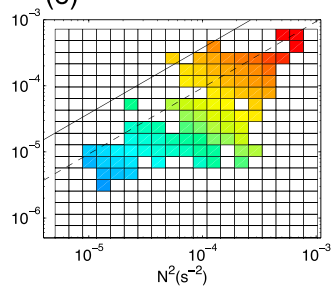

(c)
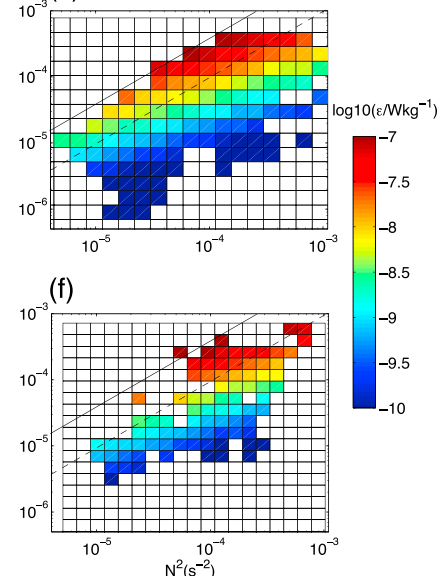

(h)
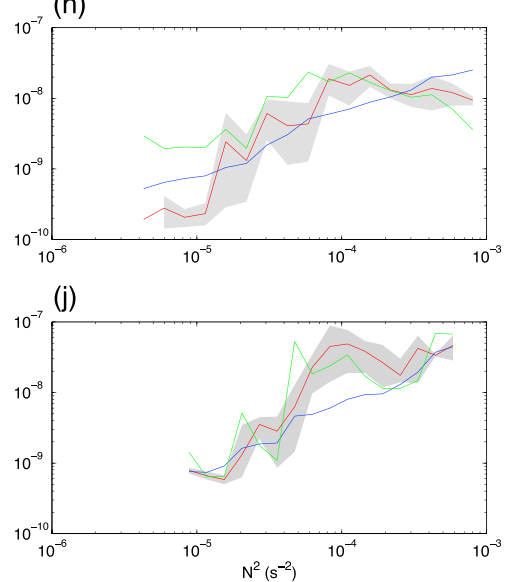

Figure 4. Distribution of dissipation rates in bins of $N^{2}$ and $S^{2}$ from (a and d) observations and the (b and e) MG and (c and f) GH parameterizations. The averaged dissipation rate in bins of (g and i) $S^{2}$ and (h and j) $N^{2}$, respectively. Figures $4 \mathrm{a}-4 \mathrm{c}, 4 \mathrm{~g}$, and $4 \mathrm{~h}$ show the observation period of VMP. Figures $4 \mathrm{~d}-4 \mathrm{f}, 4 \mathrm{i}$, and $4 \mathrm{j}$ for the observation period of SCAMP. The red, blue, and green curves in Figures $4 \mathrm{~g}-4 \mathrm{j}$ are the results of the observation, MG model, and $\mathrm{GH}$ model, respectively. The grey shading indicates $95 \%$ confidence interval. The oblique solid and dashed lines in Figures $4 \mathrm{a}-4 \mathrm{f}$ are the boundaries of $R i=0.25$ and $R i=1$.

$1.5 \times 10^{-5} \mathrm{~m}^{2} \mathrm{~s}^{-1}$, slightly larger than background values found in the open ocean thermocline. Below the thermocline, mixing becomes quite weak $\left(\varepsilon<10^{-9} \mathrm{~W} \mathrm{~kg}^{-1}, K_{\rho}<10^{-5}\right.$ $\mathrm{m}^{2} \mathrm{~s}^{-1}$ ) except for a few patches. The time-mean profiles along isotherms also display the above depth variations of $\varepsilon$ and $K_{\rho}$ (inserted panels in Figures $3 \mathrm{~b}$ and $3 \mathrm{c}$ ).

[11] In the $4 \mathrm{~m}$ shear field, a narrow band of strong shear caused by ITs is clearly identified within the thermocline with the strongest stratification (Figure 3e). Because of the large thermocline displacement caused by interfacial tides, the strong shear band is vertically elevated and depressed in a semidiurnal period. The time-averaged $S^{2}$ and $N^{2}$ show the same variation with decreasing temperature/depth (inserted panel in Figure 3d). However, $S^{2}$ is smaller than $N^{2}$, implying that the Richardson number $\operatorname{Ri}\left(=N^{2} / S^{2}\right)$ is larger than 1 and that the large-scale waves are stable toward shear instability. Since the strong stratification in the top of the thermocline (Figure $3 \mathrm{~d}$ ) inhibits the penetration of surface turbulence into the thermocline, the enhanced $\varepsilon$ inside the thermocline is likely to be associated with strong shears in the thermocline (Figure 3e). Below the thermocline, weaker $\varepsilon$ is associated with smaller shear and stratification. In addition, dissipation rates as well as $K_{\rho}$ in the troughs are elevated relative to those in the crests. This may be associated with disintegration of IT troughs into a group of ISWs that further enhances turbulent mixing (see inserted panel in Figure 3b).

\subsection{Mixing Parameterization}

[12] In order to investigate further the relationship between dissipation rates and shear/stratification, the observed dissipations rate were averaged into logarithmic bins of $N^{2}$ and $S^{2}$. Two parameterizations, MacKinnon-Gregg (MG) and Gregg-Henyey $(\mathrm{GH})$, are tested. The former was found to be relevant in situations where shear of large-scale waves and energy of small-scale waves do not maintain a particular relationship through the Garrett-Munk (GM) spectrum as illustrated for internal wave mixing in the coastal ocean [MacKinnon and Gregg, 2003], while the latter applies for internal wavefields with the GM spectral shape and is widely encountered in the open ocean [Gregg, 1989]. These two parameterizations are given by

$$
\begin{aligned}
& \varepsilon_{G H}=1.8 \times 10^{-6} f \cosh ^{-1}\left(N_{0} / f\right)\left(S / S_{\mathrm{GM}}\right)^{4}\left(N / N_{0}\right)^{2} \\
& S_{\mathrm{GM}}^{4}=1.6 \times 10^{-10}\left(N / N_{0}\right)^{2}
\end{aligned}
$$




$$
\varepsilon_{\mathrm{MG}}=\varepsilon_{0}\left(N / N_{0}\right)\left(S / S_{0}\right),
$$

where $N_{0}=S_{0}=0.0052 \mathrm{~s}^{-1}$ and $\varepsilon_{0}$ is an adjustable constant. This constant was set as $2.2 \times 10^{-9} \mathrm{~W} \mathrm{~kg}^{-1}$ using profiles without ISWs so that the mean $\varepsilon_{\mathrm{MG}}$ matches the observed mean dissipation rate. Data within the mixed layer, namely those above the thermocline $\left(T>17.3^{\circ} \mathrm{C}\right)$, were removed for the comparison. Since ADCP data in the lower layer have many gaps, only data above the depth of $110 \mathrm{~m}$ were used. Meanwhile, $150 \mathrm{kHZ}$ ADCP data were $30 \mathrm{~min}$ averaged before computing shear, as we do not focus on highfrequency shear. Stratification data were smoothed by an $8 \mathrm{~m}$ Bartlett filter to agree with the spatial response of ADCP. The dissipation rates were vertically averaged over $4 \mathrm{~m}$. Since results measured by different microstructure instruments may have bias, VMP and SCAMP data were calculated independently. The results are displayed in Figure 4.

[13] During VMP measurements, high dissipation values are encountered in strong sheared and stratified regions (Figure 4a). The distribution of the measured $\varepsilon$ in the space of $N^{2}$ and $S^{2}$ is qualitatively consistent with the MG parameterization (Figure 4b), while it is evidently different from the $\mathrm{GH}$ parameterization (Figure $4 \mathrm{c}$ ). The distribution pattern of the observed $\varepsilon$ in the space of $N^{2}$ and $S^{2}$ during SCAMP measurement is also better reproduced by the MG parameterization (Figures 3d-3f). However, in the regions of $7 \times 10^{-5} \mathrm{~s}^{-2}<N^{2}<3 \times 10^{-4} \mathrm{~s}^{-2}$, the observed $\varepsilon$ values are evidently larger than those predicted by the parameterization. This may be associated with nonlinear waves, whose mixing cannot be described by the above two parameterizations [MacKinnon and Gregg, 2003]. Note that most of ISWs appear during SCAMP measurements (see Figure 3). In addition, $R i$ is partly found to be between 0.25 and 1 , but no evident correlation between this parameter and dissipation rate is identified.

[14] To obtain the trend of dissipation rates versus $S^{2}$ and $N^{2}$ alone, the bin-sorted dissipation rates are averaged along $N^{2}$ and $S^{2}$, respectively (Figures $4 \mathrm{~g}-4 \mathrm{j}$ ). The two parameterizations show large difference in the trend of dissipation rates versus shear(Figures $4 \mathrm{~g}$ and $4 \mathrm{i}$ ), while the $\mathrm{MG}$ model during VMP measurements successfully reproduces the slope of the observed dissipation rates with increasing $S^{2}$. During SCAMP measurements, the MG model also captures the essential relationship of dissipation rate versus shear, although the dissipation rates are larger for $6 \times 10^{-6} \mathrm{~s}^{-2}<S^{2}<1 \times 10^{-4} \mathrm{~s}^{-2}$ due to the effect of nonlinear waves. Regarding the relationship between dissipation rate and stratification (Figures $4 \mathrm{~h}$ and $4 \mathrm{j}$ ), both models reflect the main variation of dissipations rates with stratification.

\section{Discussion}

[15] In the previous section, we showed that the elevated turbulent mixing in the thermocline mainly resulted from strong low-frequency (IT) shears. There large ITs do not directly cause mixing because of their stability $(R i>0.25)$, but they are likely to directly promote energy transfers toward smaller-scale waves that ultimately become unstable leading to mixing. These conditions are consistent with the simple assumption behind the MG scaling. As a result, we find that the observed dissipation rate is largely reproduced by the MG model. The large interfacial tide in the seasonal thermocline is the dominant signal throughout the central BB [Pichon, et al., 2013]. Therefore, we expect that the MG parameterization can be applied in the thermocline over the entire deep basin of the BB. The simple assumption behind the MG scaling is that the large-scale shear providing the environment for scattering and breaking of smaller waves results from a few low-frequency waves (ITs or near-inertial waves) rather than from a steady state background GM spectrum on which the GH scaling applies. This assumption is generally fulfilled in shallow coastal seas such as New England Shelf [MacKinnon and Gregg, 2003, 2005] and the Baltic Sea [van der Lee and Umlauf, 2011], where dissipations were also well parameterized by the MG model. Therefore, the MG scaling is mostly known as a shallow water parameterization. However, our observations in the deep sea that fulfill the MG assumptions, with an internal wavefield dominated by a few vertical modes at the semidiurnal frequency, show that the MG scaling has a wider range of applications than coastal seas.

[16] The large ITs that we observe in the thermocline propagate southward and mostly project on modes 3 and 4. It is quite likely that they result from scattering of IT beams generated in the northern slope of BB when beams propagate upward from the deep water and hit the thermocline following the scenario described by G01. In this course, nonlinear steepening of ITs further excites ISWs which are as well observed at a mooring location. Since the frequency of ISWs exceeds the buoyancy frequency except in the thermocline, these waves are trapped in this region where they may further increase local mixing. However because of the limited number of microstructure profiles during ISW events and the long time interval between two adjacent profiles, it was difficult to give evidence of the impact of ISWs on mixing. Additional data are required for this purpose.

[17] Acknowledgments. This work was supported by the French National Research Agency "ANR" and by CNRS (LEFE/IDAO program) in the framework of the EPIGRAM project. The authors acknowledge Theo Gerkema for useful comments on this manuscript.

[18] The Editor thanks two anonymous reviewers for their assistance in evaluating this paper.

\section{References}

Gerkema, T. (2001), Internal and interfacial tides: Beam scattering and local generation of solitary waves, J. Mar. Res., 59(2), 227-255.

Gerkema, T., F. P. A. Lam, and L. R. M. Maas (2004), Internal tides in the Bay of Biscay: Conversion rates and seasonal effects, Deep-Sea Res., 51, 2995-3008.

Gill, A. E. (1982), Atmosphere-Ocean Dynamics, pp. 662, Academic Press, Orlando, Florida.

Gregg, M. C. (1989), Scaling turbulent dissipation in the thermocline, J. Geophys. Res., 94(C7), 9686-9698, doi:10.1029/ JC094iC07p09686.

Grisouard, N., C. Staquet, and T. Gerkema (2011), Generation of internal solitary waves in a pycnocline by an internal wave beam: A numerical study, J. Fluid Mech., 676, 491-513.

Kocsis, O., H. Prandke, A. Stips, A. Simon, and A. Wuest (1999), Comparison of dissipation of turbulent kinetic energy determined from shear and temperature microstructure, J. Mar. Syst., 21, 67-84.

van der Lee, E. M., and L. Umlauf (2011), Internal wave mixing in the Baltic Sea: Near-inertial waves in the absence of tides, J. Geophys. Res., 116, C10016, doi:10.1029/2011JC007072.

MacKinnon, J. A., and M. C. Gregg (2003), Mixing on the late-summer New England Shelf-Solibores, shear, and stratification, J. Phys. Oceanogr., 33(7), 1476-1492. 


\section{XIE ET AL.: INTERNAL WAVE AND TURBULENT MIXING}

MacKinnon, J. A., and M. C. Gregg (2005), Spring mixing: Turbulence and internal waves during restratification on the New England Shelf, J. Phys. Oceanogr., 35(12), 2425-2443.

Mercier, M. J., M. Mathur, L. Gostiaux, T. Gerkema, J. M. Magalhães, J. C. B. Da Silva, and T. Dauxois (2012), Soliton generation by internal tidal beams impinging on a pycnocline: Laboratory experiments, J. Fluid Mech., 704, 37-60.

New, A. L., and J. C. B. DaSilva (2002), Remote-sensing evidence for the local generation of internal soliton packets in the central Bay of Biscay, Deep-Sea Res., 49, 915-934.

New, A. L., and R. D. Pingree (1990), Large-amplitude internal soliton packets in the central Bay of Biscay, Deep-Sea Res., 37A(3), 513-524.
Pichon, A., Y. Morel, R. Baraille, and L. S. Quaresma (2013), Internal tide interactions in the Bay of Biscay: Observations and modeling, J. Mar. Sys., 109-110, S26-S44, doi:10.1016/j.jmarsys.2011.07.003.

Pingree, R. D., and A. L. New (1991), Abyssal penetration and bottom reflection of internal tidal energy in the Bay of Biscay, J. Phys. Oceanogr., 21, 28-39.

Ruddick, B., A. Anis, and K. Thompson (2000), Maximum likelihood spectral fitting: The Batchelor spectrum, J. Atmos. Oceanic Technol., 17, 1541-1554.

Shih, L. H., J. R. Koseff, G. N. Ivey, and J. H. Ferziger (2005), Parameterization of turbulent fluxes and scales using homogenous sheared stably stratified turbulence simulations, J. Fluid Mech., 525, 193-214, doi:10.1017/S0022112004002587. 